\title{
99mTc-sestamibi scintigraphy used to evaluate tumor response to neoadjuvant chemotherapy in locally advanced breast cancer: A quantitative analysis
}

\author{
KATIA HIROMOTO KOGA ${ }^{1}$, SONIA MARTA MORIGUCHI ${ }^{5}$, JORGE NAHÁS NETO ${ }^{2}$, \\ STELA VERZINHASSE PERES ${ }^{7}$, EDUARDO TINÓIS DA SILVA ${ }^{1}$, ALMIR JOSÉ SARRI ${ }^{6}$, \\ ODAIR CARLITO MICHELIN ${ }^{3}$, MARIANGELA ESTHER ALENCAR MARQUES ${ }^{4}$ and BEATRIZ LOTUFO GRIVA $^{1}$
}

\author{
Departments of ${ }^{1}$ Nuclear Medicine, ${ }^{2}$ Obstetrics and Gynecology, ${ }^{3}$ Medical Clinic, and ${ }^{4}$ Pathology, \\ Botucatu Medical School, Sao Paulo State University (UNESP), São Paulo; Departments of ${ }^{5}$ Nuclear Medicine, \\ ${ }^{6}$ Physiotherapy, ${ }^{7}$ Center for Researcher Support, Barretos Cancer Hospital, Barretos, São Paulo, Brazil
}

Received September 16, 2009; Accepted November 30, 2009

DOI: 10.3892/ol_00000067

\begin{abstract}
To evaluate the tumor response to neoadjuvant chemotherapy, ${ }^{99 \mathrm{~m}} \mathrm{Tc}$-sestamibi breast scintigraphy was proposed as a quantitative method. Fifty-five patients with ductal carcinoma were studied. They underwent breast scintigraphy before and after neoadjuvant chemotherapy, along with clinical assessment and surgical specimen analysis. The regions of interest on the lesion and contralateral breast were identified, and the pixel counts were used to evaluate lesion uptake in relation to background radiation. The ratio of these counts before to after neoadjuvant chemotherapy was assessed. The decrease in uptake rate due to chemotherapy characterized the scintigraphy tumor response. The Kruskal-Wallis test was used to compare the mean scintigraphic tumor response and histological type. Dunn's multiple comparison test was used to detect differences between histological types. The MannWhitney test was used to compare means between quantitative and qualitative variables: scintigraphic tumor response vs. clinical response and uptake before chemotherapy vs. scintigraphic tumor response. The Spearman's test was used to correlate the quantitative variables of clinical reduction in tumor size and scintigraphic tumor response. All of the variables compared presented significant differences. The change in 99mTc-sestamibi uptake noted on breast scintigraphy, before to after neoadjuvant chemotherapy, may be used as an effective method for evaluating the response to neoadjuvant chemotherapy, since this quantification reflects the biological behavior of the tumor towards the chemotherapy regimen.
\end{abstract}

Correspondence to: Dr Katia Hiromoto Koga, Department of Nuclear Medicine, Botucatu Medical School, Sao Paulo State University (UNESP), São Paulo, Brazil

E-mail:khkoga@bol.com.br

Key words: breast cancer, neoadjuvant chemotherapy, ${ }^{99 \mathrm{~m}} \mathrm{Tc}-\mathrm{sestamibi}$, scintigraphy, quantitative analysis
Furthermore, additional analysis on the uptake rate before chemotherapy may accurately predict treatment response.

\section{Introduction}

Breast cancer is the second most common type of cancer worldwide and the most common among women. It accounts for approximately $22 \%$ of new cancer cases among women each year (1), and the 5-year survival rate among the worldwide population is $61 \%$. This high mortality rate is related to late diagnosis, with the presence of tumors of large dimensions and lymph node metastases.

Locally advanced breast cancer comprises tumors greater than $5 \mathrm{~cm}$ in diameter, with wall or skin invasion, metastases to fixed lymph nodes $(2,3)$ and inflammatory carcinoma. Treatment for this is multidisciplinary, consisting of preoperative chemotherapy, surgery, radiotherapy and postoperative chemotherapy (4-6).

Preoperative or neoadjuvant chemotherapy is standard treatment for individuals with locally advanced breast cancer (7-10). It has been found to substantially improve the survival of these patients $(6,11,12)$. It provides better local control over the disease, with increased likelihood that the breast surgery will be conservative $(4,5,13)$. Moreover, this type of chemotherapy treats preexisting microscopic systemic disease and enables the evaluation of tumor resistance in vivo (14).

Reduction in tumor volume has been used as the standard criterion for response evaluation among solid tumors such as breast carcinoma (15). Techniques that measure changes in the molecular biology of the tumor, such as Doppler ultrasonography, functional magnetic resonance and nuclear medicine are promising methods for the identification of tumors that present a favorable response, with great accuracy (16-18).

Breast scintigraphy is a well-established diagnostic imaging technique (19) of relatively low cost compared with positron emission tomography and magnetic resonance imaging. This technique can also be used as a method for evaluating the response of breast carcinoma to chemotherapy 
treatment (20), thereby providing an in vivo indication of the chemosensitivity of the tumor (8).

Based on the limitations of the clinical examination as well as the main biological characteristics of ${ }^{99 \mathrm{~m}} \mathrm{Tc}$-sestamibi uptake in malignant lesions, an evaluation of the tumor response to neoadjuvant chemotherapy treatment through quantification of ${ }^{99 \mathrm{~m} T c-s e s t a m i b i}$ was proposed using scintigrams from individuals with locally advanced breast cancer.

\section{Materials and methods}

This was a cross-sectional study conducted between 2000 and 2008. Prior approval had been obtained from the institution's research ethics committee. Fifty-five female patients with a diagnosis of locally advanced ductal invasive breast cancer were included. They underwent neoadjuvant chemotherapy and surgical complementation, with evaluation of the response to treatment through clinical measurements and scintigraphy.

Neoadjuvant chemotherapy. The patients underwent chemotherapy consisting of either the $\mathrm{AC}$ regimen (doxorubicin hydrochloride, $60 \mathrm{mg} / \mathrm{m}^{2}$ of body surface and cyclophosphamide, $600 \mathrm{mg} / \mathrm{m}^{2}$ of body surface) or the FEC regimen (5-fluorouracil, $500 \mathrm{mg} / \mathrm{m}^{2}$ of body surface; epirubicin, $60 \mathrm{mg} / \mathrm{m}^{2}$ of body surface and cyclophosphamide, $500 \mathrm{mg} / \mathrm{m}^{2}$ of body surface).

Evaluation of tumor response to neoadjuvant chemotherapy by means of clinical measurements. Tumor measurements were made by a clinical oncologist at two points: before starting and after completing the neoadjuvant chemotherapy. The size in $\mathrm{cm}$ was calculated as the mean diameter between the two largest axes. The clinical tumor response was based on the change in tumor size before and after the neoadjuvant chemotherapy, expressed as a percentage. The response was classified into two groups; tumor reduction $>60 \%$ and $<60 \%$.

${ }^{99 m}$ Tc-sestamibi breast scintigraphy. Breast scintigraphy was performed at two points: before starting and after completing the neoadjuvant chemotherapy, simultaneously with the clinical measurements of the tumor. The images were acquired by a Siemens gamma camera (Orbiter model), coupled to an Icon computer with software version 7.5 and circular detector. A foam pad of $30 \mathrm{~cm}$ in thickness was used with openings lateral to the breast projection $(21,22)$. A low-energy high-resolution collimator was used with a matrix of $128 \times 128$ pixels, a photopeak of $140 \mathrm{keV}$ and a window of $15 \%$. The image acquisition lasted for $10 \mathrm{~min}$, starting $10 \mathrm{~min}$ after injection. Posterior oblique and lateral images with magnification were obtained. The patient was in a prone position with the breasts hanging and the collimator was fitted into the openings. Anterior thoracic and axillary images without magnification were obtained with the patient in a supine position. For all of the examinations ${ }^{99 \mathrm{~m}} \mathrm{Tc}$-sestamibi was used, which has a labeling efficiency of $>95 \%$. It was administered through a vein in the foot or the arm contralateral to the compromised breast.

Quantification of ${ }^{99 \mathrm{~m}} \mathrm{Tc}$-sestamibi uptake was carried out on the lateral images of the breasts by creating two identical regions of interest: one on the tumor and the other in the mirror position on the contralateral breast. Pixel counting was performed in these regions. The tumor ${ }^{99 \mathrm{~m} T c-s e s t a m i b i}$ uptake rate was determined as the pixel count ratio between the region of interest in the tumor and the mirror region in the contralateral breast. The pretreatment $99 \mathrm{~m} \mathrm{Tc}$-sestamibi uptake rate (BUR) and post-treatment ${ }^{99 \mathrm{~m}} \mathrm{Tc}$-sestamibi uptake rate (AUR) were obtained from scintigraphic evaluations performed before starting and after completing the neoadjuvant chemotherapy, respectively. The scintigraphic tumor response (STR) was obtained from the following equation: $\mathrm{STR}=(100-\mathrm{AUR}) /(\mathrm{BUR} \times 100) \%$.

Histological evaluation. The results from the diagnostic biopsy and surgical specimen were analyzed by means of histological sections $(4-5 \mu \mathrm{m})$, cut from paraffin blocks. Hematoxylin and eosin staining was used.

Statistical analysis. To calculate the sample size a correlation coefficient (r) of 0.44 was used in the preliminary analysis. Results showed that a minimum of 45 patients would be needed. The descriptive analysis consisted of calculations of absolute and relative frequencies and central trend and dispersion measurements. The Kolmogorov-Smirnov test was used to evaluate the adherence to the normal distribution curve. Since the variables were found not to present a normal distribution, non-parametric tests were used. The Kruskal-Wallis test was used to compare the mean scintigraphic tumor response and histological type. Dunn's multiple comparison test was used to detect differences between histological types. The MannWhitney test was used to compare means between quantitative and qualitative variables: scintigraphic tumor response vs. clinical tumor response and pretreatment $99 \mathrm{mTc}$-sestamibi uptake vs. scintigraphic tumor response. The Spearman's test was used to correlate the quantitative variables of clinical reduction in tumor size and scintigraphic tumor response. The level of significance was set at $\mathrm{p}<0.05$.

\section{Results}

The patients evaluated $(\mathrm{n}=55)$ were aged between 28 and 68 years (mean 50.5; SD, 9.8; median 51). The number of cycles of chemotherapy ranged from 2 to 8 (mean 3.9; SD, 0.9; median 4). The interval between the scintigraphic examinations before starting and after completing neoadjuvant chemotherapy ranged from 2 to 7 months (mean 3.4; SD, 1.1; median 3).

Residual tumor material was observed in 49 surgical specimens, consisting of invasive ductal carcinoma in 21 cases (38.2\%) and an association between invasive ductal carcinoma and in situ ductal carcinoma in 28 cases (50.9\%). In six cases (10.9\%), the tumor response was complete. Table I shows a comparison between the mean scintigraphic tumor response and the different findings from the surgical specimen. The mean scintigraphic tumor response was found to be greatest in tumors that were reduced to zero, followed by those that were purely invasive ductal carcinomas. The mean scintigraphic tumor response was least in tumors in which there was an associated in situ component $(\mathrm{p}=0.011)$.

The ${ }^{99 m}$ Tc-sestamibi BUR ranged from 1.18 to 10.13 (mean 3.40 \pm 0.22 ), while the AUR ranged from 0.74 to 3.59 (mean 1.88 \pm 0.10 ). Thus, the overall mean scintigraphic tumor response rate was $38.31 \pm 3.26(\%)$. A higher pretreatment ${ }^{99 \mathrm{~m} T c-}$ sestamibi uptake showed greater response rates (Table II). 
Table I. Comparison between mean scintigraphic tumor response (STR) in percentages and the histological findings from the surgical specimen.

\begin{tabular}{lrcrr}
\hline Specimen & No. $(\%)$ & Mean STR\% (SD) & Median & Min-Max \\
\hline Absence of tumor & $6(10.9)$ & $63.22(16.83)$ & 71.26 & $39.05-80.86$ \\
IDC & $21(38.2)$ & $40.58(20.26)$ & 38.55 & $7.93-77.28$ \\
IDC+ISDC & $28(50.9)$ & $31.28(24.94)$ & 19.20 & $-0.83-75.61$ \\
Total & $55(100)$ & & & \\
\hline
\end{tabular}

aKruskal-Wallis test. IDC, invasive ductal carcinoma; IDC+ISDC, invasive ductal carcinoma + in situ ductal carcinoma.

Table II. Mean ${ }^{99 m}$ Tc-sestamibi uptake rate before starting neoadjuvant chemotherapy (BUR) compared with scintigraphic tumor response (STR) groups $(>60 \%$ and $<60 \%)$.

\begin{tabular}{lcccc}
\hline STR & No. $(\%)$ & Mean BUR (SD) & Median & Min-Max $^{\text {P-value }}$ \\
\hline$<60 \%$ & $42(76.4)$ & $2.82(0.96)$ & 2.55 & $1.18-5.50$ \\
$>60 \%$ & $13(23.6)$ & $5.26(2.03)$ & 5.22 & $2.54-10.13$
\end{tabular}

Total $55(100)$

${ }^{\mathrm{a} M a n n}$-Whitney test.

Table III. Mean scintigraphic tumor response (STR) in relation to groups with reduction in clinical measurement $>60 \%$ and $<60 \%$ (CTR).

\begin{tabular}{llccc}
\hline CTR & No. $(\%)$ & Mean STR\% (SD) & Median & Min-Max \\
\hline$<60 \%$ & $27(49)$ & $31.16(22.7)$ & 23.9 & $-0.8-75.6$ \\
$>60 \%$ & $28(51)$ & $45.26(23.9)$ & 48.3 & $1.3-80.8$ \\
Total & $55(100)$ & & & 0.040 \\
\hline
\end{tabular}

${ }^{\mathrm{a}}$ Mann-Whitney test. CTR, clinical tumor response.

A positive correlation was noted between the clinical and scintigraphic responses $(r=0.44 ; p=0.001)$. The scintigraphic response was greater in the tumors with a clinical response $>60 \%(\mathrm{p}=0.04)$ (Table III).

\section{Discussion}

Although clinical examinations are currently standard in evaluating tumor response to chemotherapy treatment, these measurements do not reflect the biological characteristics of tumors, nor do they reflect the real reduction in tumor size. This is because these measurements do not discriminate between inflammatory and healing processes in viable tumors.

On the other hand, the uptake characteristics and efflux dynamics of ${ }^{99 \mathrm{~m} T c-s e s t a m i b i}$ have shown a high correlation with the biological behavior of breast tumors, particularly in relation to aggressiveness and chemoresistance.

In the present study, the focus was on the relationship between the ${ }^{99 \mathrm{~m}} \mathrm{Tc}$-sestamibi uptake in locally advanced breast tumors and the histological type, and between this uptake and the clinically measured response, as a method for evaluating the response to chemotherapy treatment.

Based on the evaluation of the tumor histology in the surgical specimen, we observed that the individuals with invasive ductal carcinoma presented a greater scintigraphic tumor response than those who had an association with an in situ component. This finding may reflect the lower response of in situ carcinomas to chemotherapy, thus suggesting that calculation of the ${ }^{99 \mathrm{~m}} \mathrm{Tc}$-sestamibi uptake has additional usefulness for possible prediction of the chemotherapy response to different types of carcinomas.

With regard to comparing the pretreatment $99 \mathrm{~m} \mathrm{Tc}-\mathrm{ses}-$ tamibi uptake with the scintigraphic tumor response, Del Vecchio et al (23) obtained similar results and stated that high early ${ }^{99 \mathrm{~m}} \mathrm{Tc}$-sestamibi uptake in breast carcinomas was related to high rates of cell proliferation. This indicates greater aggressiveness of tumor behavior and correlates directly with a better and faster tumor response to chemotherapy. On the

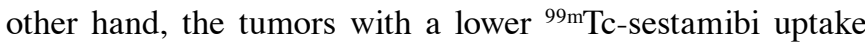
showed a lower scintigraphic response. This finding may 
be related, not only to the histological type, but also to the chemoresistance that was indicated by the inverse correlation between the intensity of ${ }^{99 \mathrm{~m} T c-s e s t a m i b i}$ uptake and Bcl-2 levels (24).

We observed that almost all of our patients presented a reduction in ${ }^{99 \mathrm{~m} T c}$-sestamibi uptake $(\mathrm{n}=54)$ after chemotherapy. Mankoff et al (20), Marshall et al (25) and Cwikla et al (26) also reported a reduction in the tumor-to-background ratio, thus showing that ${ }^{99 \mathrm{~m}} \mathrm{Tc}$-sestamibi uptake reflects the metabolic activity of the tumor and its reduction consequent to chemotherapy (25). Wilczek et al (27) found a significant reduction in the tumor-to-background ratio after the conclusion of neoadjuvant chemotherapy, which was confirmed by the tumor regression noted in the histological evaluation of the surgical specimens. These findings suggest that calculating the scintigraphic tumor response should be included as a safe parameter when evaluating tumor regression.

Quantitative analysis of breast scintigraphy using ${ }^{99 m}$ Tc-sestamibi was shown to be an additional tool for evaluating the preoperative chemotherapy response, given that the variation in ${ }^{99 \mathrm{~m} T c}$-sestamibi uptake reflects the biological behavior of the tumor. Thus, a quantitative analysis of breast scintigraphy using ${ }^{99 \mathrm{~m}} \mathrm{Tc}$-sestamibi should be included in the approach towards assessing tumor response related to preoperative chemotherapy.

\section{References}

1. Brasil. Instituto Nacional do Câncer: Estimativas 2008: Incidência de Câncer no Brasil. Instituto Nacional do Câncer, Rio de Janeiro, 2007.

2. Beahrs O: Staging of cancer. CA Cancer J Clin 41: 121-125, 1991.

3. Hortobagyi GN, Ames FC, Buzdar AU, et al: Management of stage III primary breast cancer with primary chemotherapy, surgery and radiation therapy. Cancer 62: 2507-2516, 1988.

4. Hortobagyi GN, Spanos W, Montague E, Buzdar AU, Yap HY and Blumenschein GR: Treatment of locoregionally advanced breast cancer with surgery, radiotherapy and combination chemoimmunotherapy. Int J Radiat Oncol Biol Phys 9: 643-650, 1983.

5. Hortobagyi GN: Multidisciplinary management of advanced primary and metastatic breast cancer. Cancer 74: S416-S423, 1994.

6. Hortobagyi GN, Blumenschein GR, Spanos W, Montague ED, Buzdar AU, Yap HY and Schell F: Multimodality treatment of locoregionally advanced breast cancer. Cancer 51: 763-768, 1983.

7. Booser DJ and Hortobagyi GN: Treatment of locally advanced breast cancer. Semin Oncol 19: 278-285, 1992.

8. Hortobagyi GN: Comprehensive management of locally advanced breast cancer. Cancer 66: 1387-1391, 1990.

9. Reubens RD, Bartelink H, Engelsman E, et al: Locally advanced breast cancer: the contribution of cytotoxic and endocrine treatment to radiotherapy. An EORTC breast cancer co-operative trial (10792). Eur J Cancer 25: 667-678, 1989.

10. Swain SM, Sorace RA, Bagley CS, et al: Neoadjuvant chemotherapy in the combined modality approach of locally advanced nonmetastatic breast cancer. Cancer Res 47: 3889-3894, 1987.
11. Feldman LD, Hortobaygi GN, Buzdar AU, Ames FC and Blumenschein GR: Pathological assessment of response to induction chemotherapy in breast cancer. Cancer Res 46: 2578-2581, 1986.

12. Buzdar A, Montague E, Barker J, Hortobagyi G and Blumenschein G: Management of inflammatory carcinoma of the breast with combined modality approach - an update. Cancer 47: 2537-2542, 1981

13. Smith IC, Heys SD, Hutcheon AW, et al: Neoadjuvant chemotherapy in breast cancer: significantly enhanced response with docetaxel. J Clin Oncol 20: 1456-1466, 2002.

14. Dunnwald LK, Gralow JR, Ellis GK, et al: Residual tumor uptake of [99m $\mathrm{Tc}]$-sestamibi after neoadjuvant chemotherapy for locally advanced breast carcinoma predicts survival. Cancer 103: 680-688, 2005.

15. Therasse P, Arbuck SG, Eisenhauer EA, et al: New guidelines to evaluate the response to treatment in solid tumors. J Natl Cancer Inst 92: 205-216, 2000.

16. Wahl R, Zasadny K, Halvie M, Hutchins G, Weber B and Cody R: Metabolic monitoring of breast cancer chemohormonotherapy using positron emission tomography: initial evaluation. J Clin Oncol 11: 2101-2111, 1993.

17. Abraham DC, Jones RC, Jones SE, et al: Evaluation of neoadjuvant chemotherapeutic response of locally advanced breast cancer by magnetic resonance imaging. Cancer 78: 91-100, 1996.

18. Kedar RP, Cosgrove DO, Smith IE, Mansi JL and Bamber JC: Breast carcinoma: measurement of tumor response to primary medical therapy with color flow Doppler imaging. Radiology 190: 825-830, 1994.

19. Khalkhali I, Diggles LE, Taillefer R, Vandestreek PR, Peller PJ and Abdel-Nabi HH: Procedure guideline for breast scintigraphy. J Nucl Med 40: 1233-1235, 1999.

20. Mankoff DA, Dunnwald LK, Gralow JR, Ellis GK, Drucker MJ and Livingstone RB: Monitoring the response of patients with locally advanced breast carcinoma to neoadjuvant chemotherapy using [technetium 99m]-sestamibi scintimammography. Cancer 85: 2410-2423, 1999.

21. Khalkhali I, Mena I and Jouanne E: Prone scintimammography in patients with suspicion of breast cancer. J Am Coll Surg 78: 491-497, 1994.

22. Diggles L, Mena I and Khalkhali I: Technical aspects of prone dependent-breast scintimammography. J Nucl Med Tech 22: 165-170, 1994.

23. Del Vecchio S, Zannetti A, Fonti R, Iommelli F and Salvatore M: ${ }^{99 m}$ Tc-MIBI in the evaluation of breast cancer biology. In: Breast Cancer Nuclear Medicine in Diagnosis and Therapeutic Options. Bombardieri E, Bonadonna G and Gianni L (eds). Springer, Berlin, pp71-81, 2008.

24. Del Vecchio S, Zannetti A, Aloj L, Caraco C, Ciarmiello A and Salvatore M: Inhibition of early ${ }^{99 \mathrm{~m}} \mathrm{Tc}-\mathrm{MIBI}$ uptake by Bcl-2 antiapoptotic protein overexpression in untreated breast carcinoma. Eur J Nucl Med Mol Imaging 30: 879-887, 2003.

25. Marshall C, Eremin J, Mohammed ES, Eremin O and Griffiths A: Monitoring the response of large $(>3 \mathrm{~cm})$ and locally advanced (T3-4, N0-2) breast cancer to neoadjuvant chemotherapy using 99mTc-sestamibi uptake. Nucl Med Commun 26: 9-15, 2005.

26. Cwikla JB, Buscombe JR, Barlow RV, et al: The effect of chemotherapy on the uptake of technetium- $99 \mathrm{~m}$ sestamibi in breast cancer. Eur J Nucl Med 24: 1175-1178, 1997.

27. Wilczek B, Von Schoultz E, Bergh J, Eriksson E, Larsson SA and Jacobsson H: Early assessment of neoadjuvant chemotherapy by FEC-courses of locally advanced breast cancer using ${ }^{99 \mathrm{~m} T c-M I B I}$. Acta Radiol 43: 284-287, 2003. 